\title{
Difficultés De Mise En CEuvre De La Continuité Didactique Mathématique-Mécanique Au Secondaire Collégial, Registre Sémiotique Et Transfert Comme Éléments D'analyse
}

\section{Khadija Raouf}

Enseignant chercheur et formatrice des futurs enseignants des sciences physiques,

Observatoire de recherche en didactique et pédagogie universitaire

Centre Régional des Métiers de l'Education et de la Formation d’Eljadida, Maroc

\section{Mourad Radi}

Imad Belazzaar

Enseignants des sciences physiques, cycle de l'enseignement secondaire collégial Ministère de l’Education Nationale et de la Formation

Professionnelle, Maroc

\section{Mohammed Talbi \\ Mohamed Moussetad}

Enseignants chercheurs, Observatoire de recherche en didactique et pédagogie universitaire Faculté des sciences Ben M’Sik. Hassan II University, Casablanca, Maroc

doi: 10.19044/esj.2016.v12n31p165 URL:http://dx.doi.org/10.19044/esj.2016.v12n31p165

\begin{abstract}
This article deals with the numerous pedagogical and didactic constraints to the transdisciplinary knowledge transfer between mathematics and physics (mechanics) at the last year of secondary school. By taking into consideration the three entities of the didactic triangle (Content, students and teachers), we analyzed in this exploratory study official pedagogical orientations, curricula and textbooks of mathematics and physics. Then, we administered to a sample of 12 to 15-year-old pupils, mathematical and physical written questionnaires in which they have to use proportionality/linearity concept by considering different register of semiotic representation and the rationality frame of each discipline., the analysis of the results reveal some difficulties that can be explained by a lack of knowledge related to the change of rationality frame and semiotic register rules. In order to understand the origin of these difficulties, we proceeded by analyzing the transdisciplinary practices of mathematics/physics teachers by
\end{abstract}


means of a written semi-open questionnaire whose results highlight interdidactic rupture between mathematics and physics. To remedy that, we propose some strategies and possible lines of actions.

Keywords: Transdisciplinary techniques, knowledge mobilization, transfer, semiotic registers

\section{Resume}

Cet article traite les différentes contraintes pédagogiques et didactiques à la mise en œuvre de la continuité mathématique-mécanique en dernière année du cycle du secondaire collégial. Pour ce faire, nous avons pris en considération, dans cette étude exploratoire, les trois composantes du triangle didactique. Nous avons en premier lieu procédé à l'analyse des orientations pédagogiques, des programmes et des manuels scolaires actuels des mathématiques et de la physique. Ensuite, nous avons soumis à un échantillon d'élève (12-15 ans) deux questionnaires écrits l'un en mathématiques et l'autre en mécanique dont la résolution des situations interpellent le concept proportionnalité/linéarité tout en considérant les différents registres de représentation et le cadre de rationalité propre à chaque discipline. Les résultats révèlent des difficultés liées vraisemblablement à une méconnaissance des règles de changement sémiotiques ainsi qu'au changement de cadre de rationalité. Pour comprendre l'origine de ces difficultés, nous avons opté pour l'analyse des pratiques transdisciplinaires d'un échantillon d'enseignants des deux disciplines via un questionnaire écrit semi-ouvert. Les résultats de dépouillement du questionnaire semblent indiquer des pratiques et des représentations favorisant le cloisonnement entre les deux disciplines traduit par une rupture inter-didactique mathématiques-physique. Pour y remédier, nous proposons quelques stratégies et pistes d'actions.

Mots-clés : Pratiques transdisciplinaires, mobilisation des connaissances, transfert, registres sémiotiques

\section{Introduction}

Au Maroc, l'enseignement est organisé en 3 niveaux : le préscolaire et le primaire, le secondaire et le supérieur. L'enseignement secondaire, d'une durée de 6 ans, comporte le cycle collégial et le cycle qualifiant. Le cycle secondaire collégial, d'une durée de 3 ans, est sanctionné par un brevet d'enseignement collégial (BEC) qui permet la transition au cycle secondaire qualifiant. Au collège, le programme vise le renforcement des acquis de l'enseignement primaire et le développement progressif de nouveaux apprentissages qui auront des prolongements dans le cycle du secondaire 
qualifiant. Cependant, le programme de la mécanique est enseigné pour la première fois au deuxième semestre de la dernière année du cycle.

L’apprentissage de la mécanique a fait l’objet de plusieurs recherches axées sur les conceptions erronées des élèves, comme obstacle à l'apprentissage du concept "Force", traitées par plusieurs chercheurs notamment Viennot (1986), Mohapatra et Bhattacharyya (1989), Twigger et al (1994), Brasquet (1999), Maarouf et Kouhila (2001), Koffi (2010). Quant aux difficultés d'articulation mathématiques-physique, Ba (2011) s'est intéressé aux concepts vecteurs et grandeurs physiques vectorielles. A partir des résultats d'analyse des manuels des mathématiques et de la physique de la seconde $\mathrm{S}$ au Sénégal et de la première $\mathrm{S}$ en France, l'auteur estime que les situations physiques introduites dans les manuels de mathématiques manquent de réalisme du point de vue de la problématique physique et que les objets mathématiques ne sont utilisés que comme outils. Boumghar et al (2012) ont procédé à l'analyse des manuels de physique algériens à propos du concept "Force". Ils soulèvent le caractère mathématisé de la démarche de transposition du savoir savant au savoir à enseigner dans le programme de mécanique au collège et son rôle dans la persistance des difficultés des collégiens sous-jacentes à l'enseignement-apprentissage du concept "Force".

Dans le contexte marocain, on note les travaux de Maarouf et Kouhila (2001) sur les difficultés rencontrées par les élèves lors de l'étude de situations mécaniques modélisables en termes de "Force" au collège. Les activités de modélisation en question, concernent la définition du système étudié, l'inventaire des forces qui agissent sur le système et la représentation de ces forces à l'aide des entités vectorielles. Les auteurs attribuent les difficultés d'acquisition des premières notions de mécanique principalement à la non intégration des processus de modélisation et de conceptualisation dans les structures cognitives des collégiens marocains.

"[..] les représentations symboliques des concepts de la physique sont souvent parachutées par l'enseignant sans être discutées avec les élèves. L'élève est amené à accepter des modèles tout faits sans qu'il soit impliqué dans les processus intellectuels (problématique, conceptualisation, modélisation, formalisation, etc.) qui mènent à leur élaboration. Il est tout simplement sollicité pour appliquer ces modèles afin de répondre à des questions écrites sur des situations elles mêmes décrites [..]". p 43-54

Les auteurs recommandent d'expliciter aux apprenants le mode de fonctionnement spontané et le mode de fonctionnement scientifique afin de développer chez eux le processus de pensée propres aux sciences physiques.

En plus de ces difficultés d'apprentissage de la mécanique, peut on ignorer celles sous-jacentes à la non maitrise des objets mathématiques impliqués dans la modélisation mathématique ou celles inhérentes au changement du cadre de rationalité ? 


\section{Problematique de la recherche}

Lors de l'accompagnement des futurs professeurs des sciences physiques, durant leurs stages de mise en situations professionnelles (MSP), effectués dans les établissements scolaires, nous avons constaté que certains élèves de la $3^{\text {ème }}$ année du secondaire collégial trouvent des difficultés à mobiliser leurs connaissances mathématiques dans des activités de modélisation en mécanique. Ces difficultés se manifestent, à titre d'exemple, au niveau de la transformation de la relation $V=d / t, D_{A}=D_{R}+D_{F}\left(D_{A}\right.$ : la distance d'arrêt, $\mathrm{D}_{\mathrm{R}}$ : la distance de réaction et $\mathrm{D}_{\mathrm{F}}$ : la distance de freinage) et $\mathrm{P} / \mathrm{m}=\mathrm{g}$. Cette relation de proportionnalité entre ces différentes grandeurs physiques fait appel aux fonctions cognitives d'un système sémiotique à savoir la fonction de traitement et/ou la fonction de conversion (Duval, 1993, 1995) et s’appuie sur le concept de la proportionnalité que les élèves sont sensés maitriser en cours de mathématique. Or, les résultats des enquêtes nationales relatives à l'évaluation des acquis en mathématiques, (CSEFRS, 2009), montrent que $84 \%$ des élèves de la $3^{\text {ème }}$ année et $92 \%$ de la $2^{\text {ème }}$ année du secondaire collégial ont un score bien inférieur à la moyenne (COSEFRS, 2014). Ce niveau faible des acquis de nos élèves en mathématiques a été confirmé par les résultats de l'enquête de TIMSS (1999-2011). Ces données peuvent-elles expliquer, à elles seules, les difficultés de mobilisation des notions mathématiques, susmentionnées, dans la physique en général et dans la mécanique en particulier? Les élèves maitrisent-ils les règles de changement de registres sémiotiques au sein d'un même cadre de rationalité ? Qu'en est-il dans le cas de changement de cadre de rationalité ? Comment les enseignants des deux disciplines gèrent-ils les problématiques liées au transfert transdisciplinaire induits par la modélisation mathématique? Leurs pratiques favorisent-elles la continuité mathématiques-physique?

Dans cet article, nous essayerons d’apporter des éléments de réponse à ces questions en nous appuyant sur la combinaison de deux éléments d'analyse: les registres sémiotiques au sens de Duval (1993,1995) (implicitement le cadre de rationalité (Lerouge, 2000) qui leur donne sens) et le transfert des connaissances d'une discipline à l'autre.

\section{Cadre conceptuel}

Les rapports longuement privilégiés, entre les sciences physiques et les mathématiques ont connu d'importantes mutations oscillant entre rapprochement et rupture (Henry dans Toussaint, 1996; Artaud, 1999 ; $\mathrm{Ba}, 2011)$. Ces mutations ont influencé l'évolution des deux disciplines et par conséquent les pratiques d'enseignement. Les rapports d'ordre constitutif entre les mathématiques et la physique permettent une double émergence de concepts (Robert et Treiner, 2009). En effet, la collaboration étroite entre les 
deux disciplines a été à l'origine de l'invention et du développement de plusieurs concepts, à titre d'exemple le calcul infinitésimal. Des dérivées et des différentielles inventés par Newton et Liebniz ont permis aux, mathématiques et à la physique de formuler des lois naturelles et de développer de grands modèles théoriques (Henry dans Toussaint, 1996).

A ce propos, Rutherford et Ahlgren (1990, in Prestwich, 2012) écrit: "[..] the alliance between science and mathematics has a long history, dating back centuries. Science provides mathematics with interesting problems to investigate, and mathematics provides science with powerful tools to use in analyzing data".p 16

Cette alliance procure à la modélisation le statut de "lieu privilégié" d'interaction entre les deux disciplines. Ainsi, au niveau scolaire, la construction du réel par la pensée se fait à la fois via un modèle physique et un modèle mathématique. Au collège, l'établissement des lois (loi d’Ohm) et des relations de proportionnalité entre différentes grandeurs physiques (le poids et la masse, etc.) se fait via l'activité de traitement d'un système sémiotique qui consiste à transformer la représentation au sein du même registre et/ou via l'activité de conversion dont la transformation se fait dans un registre autre que celui où elle été formée (Duval, 1993, 1995). Ces deux activités constituent à côté de celle de la formation d'une représentation identifiable comme une représentation d'un registre donné, les activités cognitives fondamentales pour qu'un système sémiotique puisse être un registre de représentation (Duval, 1993). La conversion des représentations permet d'expliciter d'autres significations relatives à ce qui est représenté (Duval, 1995). Il est important de noter que le fonctionnement véritable d'une représentation en tant que telle dépend de deux conditions (Duval, 1993) :

"[..] il faut que l'objet ne soit pas confondu avec ses représentations et qu'il soit reconnu dans chacune de ses représentations possibles. [..]" p.4

C'est dans ces conditions que la coordination entre ces différents registres et représentations sémiotiques (Numérique, écriture algébrique, représentation graphique), introduits par Duval spécifiquement en didactique des mathématiques, puisse assumer son rôle fondamental dans la compréhension conceptuelle et dans le fonctionnement cognitif de la pensée (Duval, 1993 et 1995). Cette coordination entre registres, caractérisée par la spontanéité et la rapidité de l'activité de conversion, reste difficile à concrétiser dans le cadre d'un enseignement axé principalement sur des contenus conceptuels (Duval, 1993) et dans lequel on accorde plus d'importance aux représentations mentales qu'aux représentations sémiotiques (Duval, 1988 et 1993).

Duval (1993) écrit à partir de l'analyse des résultats d'expérimentation sur un échantillon d'élèves : 
"[..]On peut observer à tous les niveaux un cloisonnement des registres de représentation chez la très grande majorité des élèves. Ceux-ci ne reconnaissent pas le même objet à travers des représentations qui en sont donnés dans des systèmes sémiotiques différents : l'écriture algébrique d'une relation et sa représentation graphique [..] ce cloisonnement subsiste même après un enseignement sur des contenus mathématiques ayant largement utilisé ces différents registres." p.52

Dans le cadre de la modélisation mathématique, il est essentiel d'associer le registre sémiotique au cadre de rationalité (Douady, 1984 et Lerouge, 1992) qui lui donne sens surtout que la démarche adoptée dans l'enseignement favorise un traitement didactique reposant sur un homomorphisme implicite entre le concept dans le cadre des mathématiques et celui dans le cadre de la physique (Malafosse et Dusseau, 2001). C'est le cas par exemple de la droite, qui dans le contexte de la physique expérimentale ne garde pas le même statut qu'en mathématique (Lerouge, 1992 ; Malafosse et al, 2000). Cette mise en relation entre le registre sémiotique et le cadre de rationalité favoriserait la mise en œuvre de la continuité didactique mathématique-physique (Malafosse et al, 2000 et Malafosse, 2002). Or cette continuité dépend des choix fondamentaux des programmes, des épistémologies sous-jacentes à chaque discipline et de leurs interprétations par les enseignants, elles même tributaires de leur propre formation (Henry, 1996). Ces choix fondamentaux affectent le processus d'apprentissage chez l'apprenant et sa capacité à mobiliser des connaissances mathématiques dans le domaine de la physique. Cette mobilisation dépend de l'étendue de la maitrise de ces connaissances dans le cadre des mathématiques (situation source), du sens que l'on donne aux apprentissages, de la compréhension de la situation cible (cadre de la physique) et du pourquoi on fait appel à une telle connaissance acquise en situation source dans une telle situation cible (Forcier et Goulet, 1996). L'aptitude au transfert dépend, certes du degré de la maîtrise des connaissances à mobiliser dans la situation source, mais aussi du degré de l'organisation et de la structuration de ces connaissances (Moffet, 1993). Ces dernières, plus elles sont organisées et structurées plus il devient aisé pour l'élève d'y avoir accès et de les utiliser à bon escient.

Ces connaissances encodées, emmagasinées et organisées seront ultérieurement rappelées et activées pour servir à l'accomplissement d'une nouvelle tâche ou un nouvel apprentissage (Presseau, 2000), d'où l'importance accordée à la façon dont le savoir a été appris dans le contexte initial d'acquisition (Tardif \& Presseau, 1998). La mobilisation de connaissances construites dans une situation source A (situation initiale d'acquisition) vers une situation B (situation cible) exige du transféreur, entre autres, l'établissement de liens entre le cadre initial d'acquisition et le 
nouveau cadre. Il s'agit d'un travail de l'esprit, qui passe par des observations, des hypothèses, des interférences, des analogies, des comparaisons et d'autres opérations cognitives et métacognitives (Perrenoud, 2000). Ceci exige la préparation des apprenants aux activités de transfert et par conséquent un traitement didactique transdisciplinaire approprié de la part des professeurs (Forcier et Goulet, 1996 ; Lauzon, 2000), surtout que la dynamique du transfert repose sur l'articulation de plusieurs sous-processus (Genter \& al, 1993; Brake, 1998 ; Tardif, 1999) que les enseignants devraient connaître. Ces pratiques favorables au transfert sont aussi, dans une large mesure, celles qui facilitent les apprentissages (Bransford \& Schwartz 1999). D’où l'intérêt de démythifier le savoir, d'expliciter les transferts souhaités et de faire pratiquer le transfert (Forcier et Goulet, 1996).

Dans cette perspective, l'enseignement-apprentissage sollicite des enseignants d'intégrer dans la planification des cours, des actions systématiques permettant à l'élève d'abstraire des connaissances d'une situation $\mathrm{X}$, de se la présenter sous forme de concept ou de procédure dans une perspective d'usage ultérieur en dehors du contexte initial d’apprentissage (Tardif 1999; Lauzon, 2000). Ces pratiques influent la probabilité que les élèves soient en mesure de mobiliser leurs connaissances construites dans la situation source vers une situation cible et par conséquent faciliterait l'apprentissage de nouveaux apprentissages. Dans ce contexte, l'enseignant doit jouer le rôle de médiateur et d'entraineur (Forcier et Goulet, 1996 ; Lauzon, 2000) et s’ouvrir sur ses collègues afin de dépasser le cloisonnement entre les disciplines. Ceci contribuerait à donner plus de sens aux apprentissages et permettrait une meilleure gestion de temps. Or, plusieurs enseignants des sciences physiques déplorent l'incapacité de leurs élèves à réutiliser d'une façon efficace et judicieuse leurs connaissances construites dans la classe de mathématiques (Tardif \& Meirieu, 1996). A ce propos, Maingain \& al (2002) soulignent que les doléances exprimées lors de conseils de classe révèlent des déficiences transdisciplinaires difficilement assumées par l'enseignant isolé. D’après les mêmes auteurs, c'est la logique disciplinaire qui domine globalement les pratiques enseignantes. Les résultats de l'enquête menée par $\mathrm{Ba}$ (2011), auprès des enseignants des mathématiques et de la physique en rapport avec les difficultés d'articulation de la notion "vecteur" entre les deux disciplines au lycée, révèlent que les enseignants sont placés dans une logique favorisant le cloisonnement entre les deux disciplines et une méconnaissances des vrais enjeux. Ce système de fonctionnement en circuit fermé ne peut être en faveur de la continuité didactique mathématiques-physique imposée par la modélisation mathématique qui exige de l'élève de passer par des étapes de transition afin d'assurer le changement de cadre de rationalité d'une discipline à l'autre, et par conséquent, l'élève se trouve tiraillé entre le triangle didactique des 
mathématiques et celui de la physique au lieu d’être placé au centre de ceuxci (Malafosse et al, 2001).

\section{Interactions mathematiques-physique dans le programme de mecanique}

Le programme de mécanique tel qu’il est décrit dans le document officiel relatif aux orientations pédagogiques des sciences physiques au collège (version mars 2015), se compose de 5 unités réparties sur un volume horaire total de 21h, 7 heures sont consacrées aux exercices et aux activités de soutien et au contrôle continu. Les interactions entre les mathématiques et la physique se manifestent au niveau de la modélisation mathématique. Le tableau ci-dessous résume les apprentissages visés par chaque unité ainsi que les notions mathématiques mobilisées dans les activités de modélisation (instructions pédagogiques, 2015; Manuels scolaires: Almofid dans les sciences physiques, Almohit dans les sciences physiques, Ouahat des sciences physiques, Almounir dans les sciences physiques) :

\begin{tabular}{|c|c|c|}
\hline Axe & Apprentissages visés & Notions mathématiques mobilisées \\
\hline $\begin{array}{l}\text { Mouvement } \\
\text { et repos }\end{array}$ & $\begin{array}{l}\text { Définition du référentiel. } \\
\text { Description du mouvement } \\
\text { à partir de la forme de sa } \\
\text { trajectoire et de l'évolution de la } \\
\text { valeur de sa vitesse. } \\
\text { Distinction entre le type de } \\
\text { trajectoire (rectiligne, circulaire, } \\
\text { curviligne) et le type du } \\
\text { mouvement (translation, } \\
\text { rotation). } \\
\text { Expression et calcul de la vitesse } \\
\text { moyenne. } \\
\text { Les différentes natures du } \\
\text { mouvement (mouvement } \\
\text { uniforme, décéléré, accéléré). } \\
\text { Définition de la relation liant la } \\
\text { vitesse à la distance d'arrêt. } \\
\text { Dangers de la vitesse et règles de } \\
\text { la sécurité routière. }\end{array}$ & 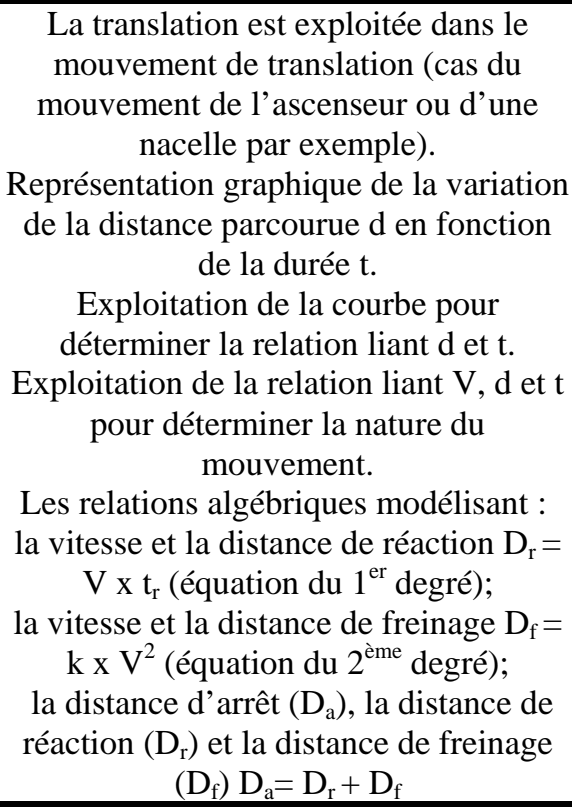 \\
\hline $\begin{array}{l}\text { Actions } \\
\text { mécaniques }\end{array}$ & $\begin{array}{c}\text { Définition des actions } \\
\text { mécaniques et leur effet. } \\
\text { Distinction entre les actions de } \\
\text { contacts et les actions à distance. }\end{array}$ & \\
\hline $\begin{array}{l}\text { Notion de } \\
\text { force }\end{array}$ & $\begin{array}{l}\text { Définition d'une force. } \\
\text { Mesure de l'intensité d'une force. } \\
\text { Représentation d'une force à } \\
\text { partir de ses caractéristiques. }\end{array}$ & $\begin{array}{l}\text { Modélisation de l'action mécanique par } \\
\text { le vecteur force : } \\
\text { Les coordonnées d'un vecteur. } \\
\text { La représentation d'un vecteur à partir } \\
\text { de ses coordonnées. }\end{array}$ \\
\hline $\begin{array}{l}\text { Equilibre } \\
\text { d'un corps }\end{array}$ & $\begin{array}{l}\text { Condition d'équilibre d'un corps } \\
\text { soumis à l'action de deux forces }\end{array}$ & $\begin{array}{c}\text { Exploitation de la définition de deux } \\
\text { vecteurs parallèles dans la modélisation }\end{array}$ \\
\hline
\end{tabular}




\begin{tabular}{|c|c|c|}
\hline $\begin{array}{c}\text { soumis à } \\
\text { l'action de } \\
\text { deux forces }\end{array}$ & et son application. & $\begin{array}{l}\text { de condition d'équilibre: } \\
\qquad \overrightarrow{\mathrm{F} 1}+\overrightarrow{\mathrm{F} 2}=\overrightarrow{\mathrm{O}}\end{array}$ \\
\hline $\begin{array}{l}\text { Poids et } \\
\text { masse }\end{array}$ & $\begin{array}{l}\text { Caractéristiques du poids d'un } \\
\text { corps solide. } \\
\text { Distinction entre poids et masse. } \\
\text { Etablissement de la relation de } \\
\text { proportionnalité entre le poids et } \\
\text { la masse ( } \mathrm{P}=\mathrm{m} \text { x g). }\end{array}$ & $\begin{array}{c}\text { La géométrie : des formes géométriques } \\
\text { (triangle, rectangle, carré, cube, } \\
\text { cylindre, etc.) sont exploitées pour } \\
\text { déterminer le centre de gravité. } \\
\text { La proportionnalité pour établir la } \\
\text { relation entre P et m. }\end{array}$ \\
\hline
\end{tabular}

Tableau 1 : Apprentissages visés et notions mathématiques mobilisées dans la modélisation

Cependant, il est recommandé aux enseignants de représenter la force modélisant les actions mécaniques par une flèche sans trop s'étaler sur le traitement mathématique de la notion "vecteur ". D’ailleurs cette consigne est respectée dans les manuels scolaires et dans les fiches pédagogiques des enseignants que nous avons consultés sur des sites web (sites mofidphysique, dorossi et adrarphysique). Concernant l'établissement de la relation de proportionnalité entre le poids d'un corps et sa masse, deux scenarios pédagogiques sont adoptés dans les pratiques enseignantes que nous avons relevés à partir de l'analyse de quatre manuels scolaires (Almofid dans les sciences physiques, Almohit dans les sciences physiques, Ouahat des sciences physiques, Almounir dans les sciences physiques) et des fiches pédagogiques disponibles sur le web. Après la mesure du poids relatif à chaque masse marquée (ou pesée), les enseignants adoptent la démarche 1 (ou 2):

\begin{tabular}{|c|c|}
\hline Démarche 1 & Démarche 2 \\
\hline $\begin{array}{l}\text { L’enseignant demande aux apprenants de : } \\
\text { Calculer le quotient P/m. } \\
\text { Comparer les différentes valeurs de P/m. } \\
\text { L'enseignant mentionne que } \mathrm{P} / \mathrm{m} \text { est } \\
\text { appelé intensité de la pesanteur qu'on note } \\
\text { g et donne sa valeur et son unité } \\
\text { La relation est établie à partir de } \mathrm{P} / \mathrm{m} \text { = g } \\
\text { NB : la pratique de la conversion du } \\
\text { registre graphique au registre algébrique } \\
\text { ou inversement se fait normalement au } \\
\text { niveau des exercices. }\end{array}$ & $\begin{array}{l}\text { L'enseignant demande aux apprenants de : } \\
\text { Calculer pour chaque mesure le quotient } \mathrm{P} / \mathrm{m} \text {. } \\
\text { Représenter la variation de } \mathrm{P} \text { en fonction de } \mathrm{m} \text {. } \\
\text { Donner la nature de la courbe (à partir de là, } \\
\text { l'élève établit que } \mathrm{P}=\mathrm{a} . \mathrm{m}) \text {. } \\
\text { Calculer le coefficient de proportionnalité et le } \\
\text { comparer avec la valeur de } \mathrm{P} / \mathrm{m} \text {, et l'élève en } \\
\text { déduit que } \mathrm{P} / \mathrm{m}=\mathrm{a} \\
\text { L'enseignant mentionne que } \mathrm{P} / \mathrm{m} \text { est appelé } \\
\text { intensité de la pesanteur qu'on note g et donne } \\
\text { sa valeur et son unité } \\
\text { La relation est établie en remplaçant a par g. }\end{array}$ \\
\hline
\end{tabular}

Tableau 2 : Démarches généralement adoptées dans l'établissement de la relation P=m.g

Si on considère la deuxième démarche, elle se base sur la coordination de la représentation numérique, de la représentation graphique et de la représentation algébrique selon le schéma suivant (Touma 2008) : 


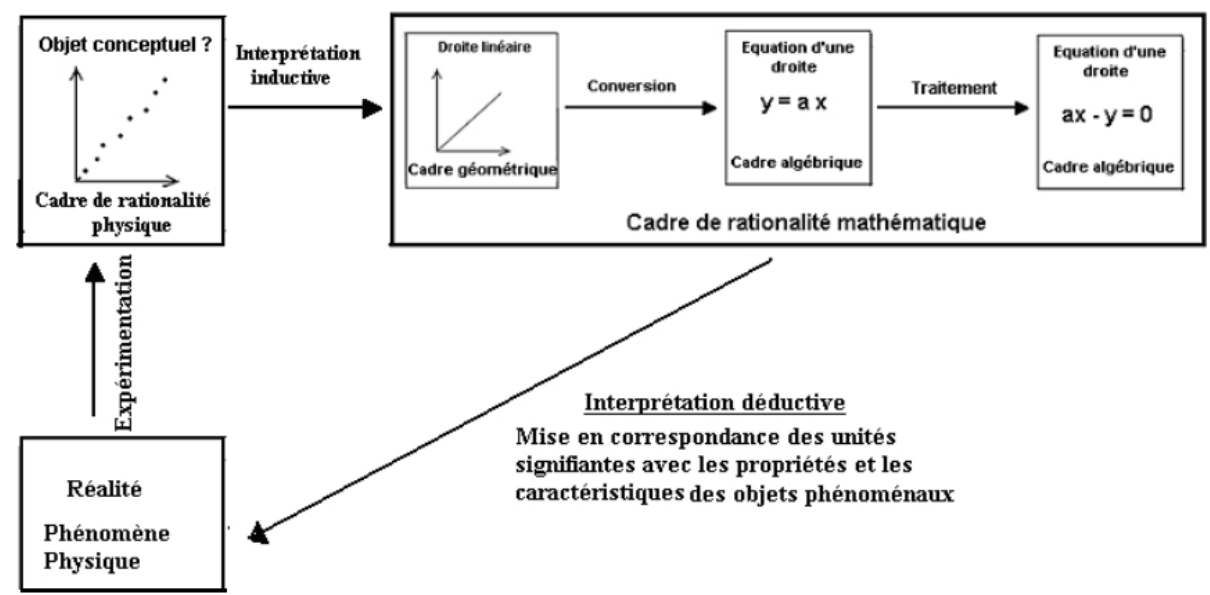

Figure1 : Registres sémiotiques et cadres de rationalité impliqués dans la démarche 2

Tiré de Touma (2008, p.106)

La stabilité de la continuité des différents registres sémiotiques, mobilisés dans le processus de modélisation mathématique, entre le cadre de la physique et le cadre des mathématiques dont la rationalité est marquée par des démarches antinomiques (Induction/déduction) (lerouge 2000, malafosse et al, 2000) interpelle le questionnement de la spécificité disciplinaire des registres sémiotiques ainsi que la mise en œuvre de stratégies d'apprentissage plaçant l'élève au cœur du triangle inter-didactique mathématiques-physique (Malafosse et al, 2000).

Dans le document officiel relatif aux orientations pédagogiques pour l'enseignement des mathématiques au cycle du secondaire collégial (Août 2009), l'interaction entre les deux disciplines est explicitée au niveau de la coordination et au niveau de l'intégration de situations physiques dans les activités de mathématiques :

" [..] en plus, l'organisation de réunions entre les enseignants des disciplines scientifiques, est une nécessité car elle permet la coordination entre ces disciplines et l'adaptation des différentes connaissances mathématiques pour une meilleure exploitation dans les autres disciplines". p.18 (Traduit de l'arabe).

Dans le tableau d'activités relatif à la $1^{\text {ère }}$ et la $2^{\text {ème }}$ année, il est suggéré d'intégrer la notion de la « vitesse moyenne » dans les contenus du programme liés à la notion " proportionnalité », par exemple.

Cependant, la divergence entre les deux cadres de rationalité des mathématiques et de la physique et la question liée à la continuité entre registres sémiotiques n'est mentionnée nulle part dans les documents officiels ni dans les guides d'enseignants ni dans le dispositif de formation des enseignants et ce pour les deux disciplines. 


\section{Methodologie de l'etude}

Afin d'explorer les différentes contraintes pédagogiques et didactiques relatives à la continuité mathématique-physique induite par la modélisation mathématique, nous avons adopté une approche mixte basée sur le questionnement des acquis d'un échantillon d'apprenants en lien avec la mobilisation du concept proportionnalité /linéarité supposé acquis dans le cours de mathématiques et des pratiques transdisciplinaires déclarées d'un échantillon d'enseignants des sciences physiques et des mathématiques. Concernant la première population cible, nous avons procédé par l'analyse d'abord des résultats du questionnaire de la mécanique afin de dégager les difficultés liées à la mobilisation des connaissances mathématiques puis des résultats du questionnaire des mathématiques. Ensuite, nous avons analysé les réponses de chaque élève relatives aux situations mécaniques et mathématiques dans le but de dégager des éléments pouvant éventuellement éclairer les trois premières questions de la recherche. Pour essayer d'apporter des éléments de réponse aux deux dernières questions de la recherche, nous avons interrogé un échantillon d'enseignants des deux disciplines sur leurs points de vue concernant les difficultés d'apprentissage disciplinaires, leurs origines éventuelles et sur leurs pratiques déclarées relatives à la gestion de la continuité didactique supposée entre les mathématiques et la mécanique.

\section{Echantillons de l'étude et outils de collecte des données Questionnaires destinés aux apprenants}

Deux questionnaires individuels écrits ont été soumis à un échantillon de 60 apprenants (12- 15 ans). L'un en mathématiques et l'autre en physique. Les situations élaborées dans les deux questionnaires sont de structure isomorphe et portent sur le concept de proportionnalité/linéarité en considérant l'articulation des registres sémiotiques et la notion de changement de cadre de rationalité. En physique, nous avons choisi comme support l'établissement de la relation entre le poids d'un corps et sa masse.

La première situation concerne l'articulation entre registre de représentation numérique et registre graphique où l'apprenant, sujet de l'expérimentation, est appelé à tracer, à partir de données consignées dans un tableau (registre numérique), la courbe représentant $\mathrm{y}$ en fonction de $\mathrm{x}$ en mathématique et le poids $\mathrm{P}$ en fonction de la masse $\mathrm{m}$ dans le cas de la mécanique (registre graphique). La deuxième situation porte sur l'articulation entre registre graphique et registre algébrique où le sujet est amené à établir, à partir du graphique tracé, la relation algébrique liant x et y en mathématique et $\mathrm{P}$ et $\mathrm{m}$ en mécanique. 


\section{Questionnaires destinés aux enseignants des sciences physiques et des mathématiques}

Dans cette étude, nous avons ciblé des enseignants expérimentés exerçant depuis 20 années et plus. En premier temps, nous avons élaboré un questionnaire écrit semi-ouvert que nous avons soumis à 100 enseignants des sciences physiques. Le taux de retour est de 65\%. Ensuite, nous avons conçu, à partir des résultats de dépouillement de ce dernier, le questionnaire semiouvert destiné à 75 enseignants des mathématiques dont le taux de retour représente $52 \%$.

Les deux questionnaires portent sur les notions à caractère disciplinaire qui présentent des difficultés d'apprentissage pour les élèves et leurs raisons d'être ainsi que sur les pratiques transdisciplinaires liées à la gestion des connaissances mathématiques mobilisées dans le contexte de la mécanique et au travail de coordination et de concertation avec les collègues de l'autre discipline :

\begin{tabular}{|c|c|c|}
\hline & \\
\hline & & Objet / Objectif \\
\hline \multirow{3}{*}{$\begin{array}{l}\text { Questionnaire } \\
\text { de mécanique }\end{array}$} & $\begin{array}{l}\text { Bloc } 1 \\
4 \text { items }\end{array}$ & $\begin{array}{l}\text { Les difficultés liées à l’apprentissage de la mécanique ainsi que les } \\
\text { hypothèses explicatives plausibles de ces difficultés. / Amorcer } \\
\text { éventuellement la problématique liée à la modélisation. }\end{array}$ \\
\hline & $\begin{array}{l}\text { Bloc } 2 \\
2 \text { items }\end{array}$ & $\begin{array}{l}\text { Les notions mathématiques que les enseignants considèrent comme } \\
\text { obstacles à l’apprentissage de la mécanique ainsi que leur point de } \\
\text { vue sur l’origine de ces obstacles. / Ces deux premiers blocs } \\
\text { pourraient nous renseigner sur les difficultés des élèves à mobiliser } \\
\text { les connaissances mathématiques en mécanique du point de vue des } \\
\text { enseignants. }\end{array}$ \\
\hline & $\begin{array}{l}\text { Bloc } 3 \\
4 \text { items }\end{array}$ & $\begin{array}{l}\text { Les pratiques enseignantes déclarées en lien avec les stratégies } \\
\text { adoptées pour palier aux dites difficultés et la nature de leurs rapports } \\
\text { professionnels avec leurs collègues de mathématiques. / Les pratiques } \\
\text { transdisciplinaires en faveur d'une rupture ou continuité didactique? }\end{array}$ \\
\hline \multirow{3}{*}{$\begin{array}{l}\text { Questionnaire } \\
\text { des } \\
\text { mathématiques }\end{array}$} & $\begin{array}{l}\text { Bloc } 1 \\
2 \text { items }\end{array}$ & $\begin{array}{l}\text { Les notions mathématiques qui présentent, selon les enseignants, des } \\
\text { difficultés majeures pour les élèves ainsi que leurs origines } \\
\text { éventuelles. / Confronter les résultats avec ceux du bloc } 2 \text { ci-dessus. }\end{array}$ \\
\hline & $\begin{array}{l}\text { Bloc } 2 \\
3 \text { items }\end{array}$ & $\begin{array}{l}\text { Les notions mécaniques mobilisant des notions mathématiques et les } \\
\text { origines éventuelles des difficultés que rencontrent les élèves dans la } \\
\text { mobilisation de ces connaissances en mécanique. / Evaluer le degré } \\
\text { de reconnaissance du programme de la mécanique par les enseignants } \\
\text { des mathématiques et dégager leurs points de vue relatives aux } \\
\text { difficultés de mobilisation de ces connaissances par les élèves en } \\
\text { dehors de leur contexte d'acquisition initial. / Les résultats de ce bloc } \\
\text { amorceraient les questions développées dans le bloc suivant. }\end{array}$ \\
\hline & $\begin{array}{l}\text { Bloc } 3 \\
4 \text { items }\end{array}$ & $\begin{array}{l}\text { Les pratiques enseignantes relatives d'une part, à la préparation des } \\
\text { élèves à la réutilisation des connaissances mathématiques dans des } \\
\text { situations autres que celles où elles ont été construites et d’autre part, } \\
\text { aux rapports de collaboration et d'échange avec les enseignants des } \\
\text { sciences physiques en lien avec la gestion des connaissances } \\
\text { mathématiques dans les deux disciplines. / les pratiques } \\
\text { transdisciplinaires en faveur d'une rupture ou continuité didactique? }\end{array}$ \\
\hline
\end{tabular}

Tableau 3 : Structure des questionnaires adressés aux enseignants des deux disciplines 


\section{Resultats et discussion}

L'analyse des résultats de dépouillement du questionnaire des mathématiques révèle que $77 \%$ des apprenants, sujets de l'expérimentation, n'ont eu aucune difficulté à représenter la courbe $\mathrm{y}=\mathrm{f}(\mathrm{x})$ à partir des données consignées dans le tableau alors que plus des deux tiers n'ont pas réussi l'articulation entre le registre de la représentation graphique et celui de l'écriture algébrique bien que les fonctions affines ont déjà été traitées aux cours des mathématiques. Ces résultats peuvent s'expliquer par le fait que les sujets ne semblent pas maîtriser les règles de correspondances sémiotique entre le registre des représentations graphiques et celui de l'écriture algébrique qui exigent la discrimination des unités significatives propres à chaque registre de représentation ainsi que l'examen des transformations implicites éventuelles requises pour changer de registre (Duval, 1988). Il se peut que dans les pratiques enseignantes, on donne plus d'importance au passage d'une représentation symbolique ou algébrique à la représentation graphique via la démarche de pointage alors que c'est le passage inverse qui pose problème (Duval, 1988). N'est-il pas plus judicieux dans l'enseignement-apprentissage des mathématiques, comme le préconise l'auteur, de passer de la logique de pointage à la logique d'interprétation globale qui interpelle plutôt l'association "variable visuelle de la représentation-unité significative de l'écriture algébrique". Cette approche de traitement global permettra éventuellement de dépasser l'obstacle de continuité du registre graphique et du registre algébrique.

En mécanique, les deux tiers des sujets n'ont pas pu représenter graphiquement la variation de $\mathrm{P}$ en fonction de la masse $\mathrm{m}$ à partir des valeurs expérimentales consignées dans le tableau. La majorité des élèves ayant réussi le test en mathématiques, n'ont pas pu faire de même en physique. Ces mêmes résultats ont été constatés pour des activités de traitement qui sollicitent des transformations au sein d'un même registre (Raouf et al, 2016). Les sujets semblent, comme dans le cas de la conversion, ne pas être préparés aux activités de transfert ni au changement de cadre. On note, dans le cas de la présente étude, que $35 \%$ des sujets ont procédé à l'association "un couple de valeurs-un point" mais n'ont pas pu tracer la droite. On pourrait penser que ces derniers ne se sont pas permis de le faire à cause éventuellement de l'obstacle de règles de raisonnement entre le cadre de rationalité des mathématiques et celui de la physique où le statut de la droite n'est pas le même (Lerouge, 2000, Malafosse, 2000 ; 2001; Malafosse et al, 2001 ; 2002).

Concernant la conversion du registre graphique au registre algébrique, $80 \%$ des sujets n'ont pas pu donner la relation de proportionnalité liant le poids $\mathrm{P}$ à la masse $\mathrm{m}$ à partir du graphique représentant la variation de $\mathrm{P}$ en fonction de $\mathrm{m}$. On note que seulement 3 
sujets sur 13 ont justifié leur réponse et le reste s'est contenté d’écrire $\mathrm{P}=$ m.g. Ces résultats pourraient vraisemblablement s'expliquer par le fait que d’une part, les apprenants sont souvent habitués à déduire la relation entre ces deux grandeurs physiques généralement à partir du calcul du quotient $\mathrm{P} / \mathrm{m}$ (qui permet d'introduire l'intensité de la pesanteur) sans passer par l'articulation registre graphique-registre algébrique et d'autre part, la coordination entre les deux registres ne semble pas pouvoir se faire sans la maitrise préalable des règles de correspondance sémiotique en mathématique. Cet ancrage dans le contexte initial d'acquisition lui confère une première stabilisation avant de songer à sa réutilisation en dehors de son contexte d'acquisition.

A priori, la conversion du registre numérique au registre graphique ne semble pas poser problème pour la majorité des sujets dans le cas des mathématiques. Cependant, dès qu'on passe du contexte des mathématiques au contexte de la physique, où la démarche de modélisation mobilise le concept de proportionnalité/linéarité, et par conséquent, invite à un changement de cadre de rationalité, la majorité des apprenants n’arrivent pas à représenter la variation de $\mathrm{P}$ en fonction de $\mathrm{m}$. En physique, ce passage d'une représentation concrète (valeurs expérimentales) à une représentation mathématiques exige des élèves des étapes de transition (Malonga, 2008 et 2009) auxquelles ils ne semblent pas être préparés. D’ailleurs, Rey et Develay (1996) précisent que le fait d’espérer qu’un élève qui sait résoudre un problème dans une situation donnée soit capable de résoudre un autre problème de même structure logique, c’est présupposer que l'identité de cette structure devrait agir comme un stimulus. La précision des similitudes et/ou des différences entre les deux situations en question, exige de l'élève la mise en œuvre de stratégies cognitives afférentes aux processus de la dynamique de transfert que nos sujets semblent ignorer. Meirieu (1991) lie l'utilisation pertinente d'un outil cognitif à la capacité du sujet à l'associer à des situations dans lesquelles il aura identifié un problème pour lequel il connait son efficacité. Comme souligné par Forcier et Goulet (1996), il ne suffit pas que les enseignants des mathématiques disent et répètent aux élèves que telle connaissance (notions, procédures, etc.) abordée en mathématiques leur servirait en mécanique, mais il faut aussi qu’ils comprennent pourquoi mobiliseraient-ils telle connaissance dans la résolution d'un problème de physique et quels sont les enjeux.

Les résultats de dépouillement des deux tests révèlent que nos sujets semblent ignorer les règles de changement de registres au sein d'un même cadre de rationalité en particulier l'articulation registre graphique-registre algébrique ainsi que celles relatives au raisonnement entre le cadre de rationalité de la physique et celui des mathématiques. A ce sujet, Duval écrit (2006) : 
"[..] Le problème cognitif que pose cette diversité de représentations possibles est celui de la reconnaissance du même something else (ou aliud aliquid), c'est-à-dire du même objet, dans les contenus différents de chacune de ses multiples représentations possibles." p.64

Ces obstacles de non continuité des registres sémiotiques au sein d'un même cadre de rationalité et entre les deux cadres de rationalité entraveraient inévitablement le transfert des connaissances mathématiques dans la modélisation mécanique. Ces résultats interpellent les pratiques transdisciplinaires relatives à l'articulation des interventions des enseignants des deux disciplines et à la planification des apprentissages en question.

Dans la suite de cette étude, nous nous intéressons aux pratiques transdisciplinaires déclarées des enseignants des sciences physiques et des mathématiques relatives à la gestion des connaissances mathématiques mobilisées dans les activités de modélisation mécaniques ainsi que leur point de vue sur les difficultés à caractère disciplinaire.

\section{Questionnaires écrits destinés aux enseignants des deux disciplines Difficultés d'apprentissage en mécanique}

Les résultats de dépouillement des réponses des enseignants des sciences physiques relatives aux notions qui présentent le plus de difficultés pour les élèves dans l'apprentissage de la mécanique sont consignés dans le tableau qui suit. Ces difficultés sont classées par ordre décroissant de la fréquence des réponses.

\begin{tabular}{|c|c|}
\hline Nature des difficultés & Fréquence \\
\hline Caractéristiques d'une force & 58 \\
\hline Représentation d'une force & 48 \\
\hline Exploitation des conditions d'équilibre d'un solide soumis à deux & 36 \\
forces & 29 \\
\hline Calcul de la vitesse d'un corps solide & 21 \\
\hline Condition d'équilibre d'un corps soumis à 2 forces & 12 \\
\hline Notion d'un corps au repos & 10 \\
\hline Typologie des actions & 8 \\
\hline Distinction entre poids et masse & 7 \\
\hline Nature du mouvement &
\end{tabular}

Tableau 4 : Les notions mécaniques présentant des difficultés d’apprentissage selon les enseignants

Quant à l'origine de ces difficultés d'apprentissage, les enseignants des sciences physiques interrogés les imputent aux éléments suivants : 


\begin{tabular}{|c|c|c|}
\cline { 2 - 3 } \multicolumn{1}{c|}{} & Fréquence & Pourcentage \\
\hline Aspect abstrait des concepts & 65 & 100 \\
\hline Manque de moyens didactiques & 65 & 100 \\
\hline $\begin{array}{c}\text { Niveau des élèves en mathématique ou leur } \\
\text { incapacité à réutiliser leurs acquis mathématiques } \\
\text { dans des situations d'apprentissage liées à la } \\
\text { modélisation physico-mathématiques }\end{array}$ & 50 & 77 \\
\hline $\begin{array}{c}\text { Programmation pédagogique de la mécanique qu'en } \\
\text { dernière année du cycle du secondaire collégial }\end{array}$ & 64 & 99 \\
\hline $\begin{array}{c}\text { Insuffisance du volume horaire destiné à } \\
\text { l'apprentissage de la mécanique }\end{array}$ & 62 & 96 \\
\hline
\end{tabular}

Tableau 5 : Origine des difficultés d'apprentissage de la mécanique selon les enseignants

La majorité des enseignants des sciences physiques estiment qu'en plus de l'aspect abstrait des concepts traités en mécanique, le niveau bas des élèves en mathématique ou leur incapacité à réutiliser leurs acquis mathématiques dans des situations d'apprentissage liées à la modélisation physico-mathématiques entravent l'apprentissage en physique. Concernant les lacunes en mathématiques, les enseignants les situent au niveau de la notion du vecteur (citée 39 fois ; 100\%), le calcul algébrique (citée 39 fois ; 60\%), les fonctions (citée 30 fois ; 46\%) et la translation (citée 10 fois ; $15 \%)$. On note que $45 \%$ des enseignants évoquent les obstacles induits par le passage des mathématiques à la physique qui se matérialisent, selon leurs déclarations, par la résistance des élèves à admettre qu'il s'agit bien de la même notion abordée en mathématique. Cette résistance de passer du cadre des mathématiques au cadre de la physique ne reflète-elle pas, entres autres, des pratiques mono-disciplinaires au détriment de pratiques prenant en compte le caractère transférable des connaissances à mobiliser lors de leur construction éventuellement dans le contexte initial d'apprentissage ?

\section{Difficultés d'apprentissage des mathématiques du point de vue des enseignants des mathématiques}

A la question ouverte relative aux notions mathématiques qui présentent des difficultés pour les élèves, la quasi-totalité des enseignants interrogés ont cité la géométrie spatiale, la translation, les vecteurs et la proportionnalité. De leur point de vue, ces difficultés d'apprentissage sont dues principalement au niveau faible des élèves qui n'ont pas ou peu acquis les soubassements nécessaires au cycle primaire. Ces déclarations sont corroborées par les résultats de l'étude réalisée dans le cadre du Programme National d'Evaluation des Acquis Scolaires de l'enseignement (CSEFRS, 2009) et ceux de TIMSS (1999-2011).

L’analyse des résultats de dépouillement de cette première partie des questionnaires écrits adressés aux enseignants des deux disciplines 
révèleraient des difficultés d'apprentissage en mécanique couvrant toutes les unités du programme à savoir «Mouvement et repos", "Actions mécaniques ", "Concept Force », "Equilibre d'un corps soumis à deux forces " et " Poids et masse ». La majorité des enseignants des sciences physiques citent la problématique de mobilisation des mathématiques comme facteur accentuant les difficultés d'apprentissage en mécanique. Le croisement des réponses des enseignants des deux disciplines relatives aux notions mathématiques dont l'apprentissage présente des difficultés pour les élèves, révèle que les notions citées sont celles qui interviennent dans le processus de modélisation en mécanique (modélisation de la relation liant la vitesse, la distance parcourue et la durée d'un corps solide, modélisation des actions mécaniques et de leurs effets, etc.).

Dans un tel environnement, comment les enseignants des sciences physiques gèrent-ils les difficultés de mobilisation de ces connaissances censées être acquises aux cours des mathématiques? Les pratiques enseignantes assurent-elles la continuité didactique mathématique-physique ?

\section{Pratiques transdisciplinaires}

Tous les enseignants des sciences physiques interrogés, sans exception, déclarent n’avoir jamais coordonné avec leurs collègues de mathématiques. Ils justifient ceci par l'absence de planification officielle des séances de coordination (100\%) et par le décalage entre leur emploi du temps et celui de leurs collègues (94\%). En effet, la problématique du type structurel, liée l'organisation du travail, constitue l'une des barrières à un enseignement axé sur le transfert des apprentissages (Tardif, 1999). De même, $100 \%$ des enseignants des mathématiques indiquent ne pas coordonner avec leurs homologues des sciences physiques. Ce problème de coordination a été abordé par Artigue et al (2009) qui attestent que les interactions des enseignants des mathématiques avec leurs collègues des autres disciplines sont limitées, notamment en France. La majorité des enseignants auxquels nous avons soumis le questionnaire, estime que c'est à l'enseignant de physique de solliciter l'enseignant des mathématiques! Cette déresponsabilisation déclarée pourrait éventuellement s'expliquer par les propos d'Artigue et al (2009) qui postulent que très souvent, de par leur formation, les enseignants de mathématiques sont peu préparés à la nécessité de la connexion de l'enseignement des mathématiques aux autres disciplines et qu'ils n'ont aucune expérience d'un réel travail de modélisation mathématique. Ces résultats sont en concordance avec les déclarations des enseignants des mathématiques relatives à l'introduction d'activités stimulant la pratique du transfert par les élèves et que nous résumons dans le tableau ci-dessous : 


$\begin{array}{ccccc}\text { Question } & \text { oui } & \text { non } & \begin{array}{c}\text { Sans } \\ \text { réponse }\end{array} & \text { Explication } \\ \begin{array}{c}\text { Mention des liens existant avec la } \\ \text { physique. }\end{array} & 21 \% & 72 \% & 7 \% & \begin{array}{c}32 \% \text { estiment que c'est au } \\ \text { professeur des sciences } \\ \text { physiques de le faire. } \\ \text { Manque de temps. }\end{array} \\ \begin{array}{c}\text { Intégration de la mécanique dans } \\ \text { des situations problème ou des } \\ \text { exercices d'application. }\end{array} & 0 \% & 100 \% & 0 \% & \begin{array}{c}\text { Ce genre d'activités n'est } \\ \text { pas mentionné dans les } \\ \text { curricula et les manuels } \\ \text { scolaires. }\end{array} \\ \begin{array}{c}\text { Consultation du programme de la } \\ \text { physique. }\end{array} & 3 \% & 95 \% & 2 \% & \end{array}$

Tableau 6 : Pratiques des enseignants des mathématiques relatives à la préparation des élèves au transfert

A la question relative à l'introduction de situations de mécanique dans l'apprentissage des mathématiques, la majorité des enseignants des mathématiques estime que les élèves ont "déjà" des difficultés à comprendre les mathématiques et par conséquent des situations de physique compliquerait davantage la tâche. A ce titre, l'un des enseignants a écrit : "je n'arrive pas à leur faire comprendre les mathématiques et vous voulez que je leur ajoute en plus la physique !".

Dans ce contexte, les enseignants des sciences physiques déclarent remédier aux difficultés liées à la modélisation mathématique par un rappel des notions mathématiques à mobiliser suivi d'exercices d'application. Ils estiment que cette opération, leur fait perdre du temps surtout que le volume horaire est insuffisant pour dispenser un programme de mécanique déjà condensé (14h à raison d'une séance de $2 \mathrm{~h}$ par semaine).

Ces pratiques déclarées traduisant un cloisonnement entre les deux disciplines ne sont-elles pas en faveur de pratiques mono-disciplinaires limitant toute possibilité de transfert des connaissances acquises en mathématiques dans le contexte de la physique?

L'attitude contractuelle adoptée par les élèves réfutant tout lien entre les acquis réalisés aux cours des mathématiques et les connaissances à mobiliser en physique s'expliquerait par l'absence d'une approche interdidactique dans le processus de construction des connaissances. La position des enseignants des mathématiques relative aux activités stimulant la préparation des élèves au transfert de leurs connaissances, accentuée par les lacunes des élèves en mathématiques ne rendraient-elles pas la tâche plus difficile aux enseignants des sciences physiques?

\section{Conclusion}

L'analyse et l'interprétation des résultats de cette étude ont permis d'une part, d'identifier les difficultés liées à la mobilisation de connaissances 
mathématiques dans des activités de modélisation mécanique qui invitent à l'articulation de registres sémiotiques et le changement de cadre de rationalité et d’autre part, de dégager, à partir des pratiques déclarées, les éléments pouvant éventuellement expliquer les difficultés de mise en œuvre de la continuité didactique mathématique-physique. Ces résultats reflètent vraisemblablement des pratiques enseignantes en faveur d'une rupture de continuité didactique mathématique-physique. Ceci pourrait s'expliquer par le fait que la formation des enseignants, de courte durée (une année) est purement disciplinaire et par les contraintes organisationnelles citées par les enseignants des deux disciplines ainsi que par la structure des programmes conçus par des concepteurs issus d'une même discipline. Certes dans les manuels, les liens transversaux avec les autres disciplines sont souvent répertoriés dans la première page de chaque unité du programme, mais en absence d'orientations officielles claires et explicites, d'encadrement de proximité et de programmes conçus par des équipes interdisciplinaires, ces liens sont peu ou jamais exploités. Il s’avère nécessaire qu’à côté de la formation sur la modélisation et le processus de conceptualisation dans les deux disciplines, d'intégrer dans la formation et l'encadrement pédagogique des enseignants des mathématiques et des sciences physiques, les processus de la dynamique du transfert et les stratégies cognitives à mettre en œuvre par les élèves dans l'apprentissage du transfert afin que ces derniers puissent mobiliser leurs apprentissages en dehors du contexte de construction initial (Tardif, 1999).

A partir de ces constats, de nos expériences de terrain et en harmonie avec les objectifs de la stratégie 2015-2030 visant le développement de la formation et de l'éducation au Maroc, il semble important de reconsidérer au niveau de l'encadrement pédagogique des enseignants, de la formation initiale et continue ainsi que les curricula, le processus de conceptualisation dans les deux disciplines ainsi que la modélisation mathématique et le paramètre du transfert au sein d'une discipline et d'une discipline à l'autre. A ce sujet, ne faudrait-il pas, comme le recommande Rogalski (2013), équilibrer dans l'enseignement des mathématiques, les mathématiques pour elles-mêmes et les mathématiques pour modéliser le réel dans une perspective interdisciplinaire tenant en compte les spécificités de chaque discipline et son rôle dans le processus de modélisation.

A cours terme, nous proposons de commencer par :

- l'organisation de séminaires d'approches transdisciplinaires au profit d'équipes polyvalentes et mixtes composées de formateurs, d'inspecteurs et de conseillers pédagogiques à élargir aux tuteurs des stages de mise en situations professionnelles tout en respectant le mode de pensée de chacune des disciplines ; 
- l'initiation des futurs enseignants des sciences physiques et des mathématiques ainsi que les enseignants en exercice, aux logiques des deux disciplines et de les outiller pour dégager ensemble les points de convergences entre les deux disciplines (Hasni et al, 2012). La modélisation mathématique pourrait être l'une des voies à explorer (Squalli et al, 2010).

Le module visant le complément de formation disciplinaire, les séances consacrées au micro-enseignement ainsi que les séances de préparation des stages de mise en situations professionnelles (MSP) et les séances de régulation peuvent être une opportunité à saisir pour faire vivre aux futurs enseignants des expériences de modélisation et de leur analyse réflexive (Artigue et al, 2009). Pour les enseignants en exercice, les rencontres avec les inspecteurs et les conseillés pédagogiques et la programmation des formations continues devraient porter aussi sur les activités sus mentionnées.

\section{References:}

1. Artaud, M. (1999). Conditions et contraintes de l'existence des mathématiques dans l'enseignement général. Permanences et évolutions. Petit x, 50, 23-38

2. Artigue, M., Dartois, Y., Pouyanne, N et Rumelhard, G. (2009). Modélisation et interactions entre mathématiques et biologie : l'expérience du Master professionnel "Didactique » à l'université Paris-Diderot-Paris 7. In, Ouvrier-Buffet, C. et Perrin Glorian, M.J. (éd.) Approches plurielles en didactique des mathématiques. Laboratoire de didactique André Revue, Université Paris Diderot. 277-293.

3. Вa, CISSÉ. (2011). Vecteur au lycée: difficile articulation entre mathématiques et physiques. Nouveaux cahiers de la recherche en éducation, 14(1), 71-83.

4. Boumghar, S., KENDIL, D., GHEDJGHOUDJ, S et LOUNIS, A. (2012). Enseignement -apprentissage du concept "Force" et persistance des difficultés : Quelle influence mathématique ?.Review of Science, Mathematics and Ict Education, 6(2), 63-81.

5. Bracke, D. (1998). Vers un modèle théorique du transfert: les contraintes à respecter. Revue des sciences de l'éducation, 24(2), 235-266.

6. Bransford, J.-D et Schwartz, D-L. (1999). Rethinking transfer: A simple proposal with multiple implications. Review in Education, 24, 61-100.

7. Brasquet, M. (1999). Du côté de la recherche en didactique. Actions, interactions et schématisation. Bulletin de l'Union des physiciens. 816.1220-1236. 
8. CSEFRS. (2009). Conseil Supérieur de l'Education et de la Formation et de la Recherche Scientifique (Royaume du Maroc). Instance nationale d'évaluation du système d'éducation formation. Rapport thématique sur les résultats du programme national d'évaluation des acquis PNEA 2008

9. CSEFRS. (2014). Conseil Supérieur de l'Education et de la Formation et de la Recherche Scientifique (Royaume du Maroc). Instance nationale d'évaluation du système d'éducation formation. Rapport analytique sur la mise en œuvre de la charte nationale d'éducation et de formation 2000-2013 Acquis, déficits et défis.

10. Douady, R. (1984). Jeux de cadres et dialectique outil-objet. Thèse d’état, Université Paris VII.

11. Duval, R. (1988). Graphiques et équations : L'articulation des deux registres. Annales de Didactique et de Sciences Cognitives, 1, 235253.

12. Duval, R. (1993). Registres de représentation sémiotique et fonctionnement cognitif de la pensée. Annales de didactique et des sciences cognitives, 5, 37-65.

13. Duval, R. (1995a). Sémiosis et pensée humaine: registres sémiotiques et apprentissages intellectuels. Berne : Peter Lang

14. Duval, R. (1995b). Quel cognitif retenir en didactique des mathématiques? Actes de VIII ${ }^{\mathrm{e}}$ École d’été de Didactique des Mathématiques.198-214

15. Duval, 2006. Quelle sémiotique pour l'analyse de l'activité et des productions mathématiques. Relime, Numéro spécial, 45-81.

16. Forcier, $\mathrm{P}$ et Goulet, J.P. (1996). Un problème et un mystère : le transfert des apprentissages, Pédagogie collégiale, 10(2), 30-32.

17. Hasni, A., Bousadra, F et Poulin, J.E. (2012). Les liens interdisciplinaires vus par des enseignants de sciences et technologies et de mathématiques du secondaire au Québec. RDST, 5, 131-156.

18. Henry, M. (1996). Physique-mathématique, mariage d'amour ou mariage de raison? -Epistémologie, rapport au savoir et contrats didactiques. In Toussaint, Didactique Appliquée de la PhysiqueChimie. NATHAN pédagogie.

19. Genter, D., Ratterman, M.J et Forbus, K.D. (1993). The role of similarity in transfer: Separating rettrievability from inferential soundness. Cognitive Pshychology, 25, 524-575.

20. Koffi, K.I.(2010). Les conceptualisations des élèves ivoiriens concernant les interactions mécaniques en physique. Revue Africaine 
de Didactique des Sciences et des Mathématiques. $\mathrm{N}^{\circ} 6$. Disponible en ligne à http://www.radisma.info/document.php?id=1021

21. Lauzon, F. (2000). Comment aider les élèves à intégrer et à transférer leurs apprentissages ? Proposition d'un itinéraire en cinq actions-réflexions. Pédagogie collégiale, 14(2), 34-40.

22. Lerouge, A. (1992). Représentation cartésienne, rationalité mathématique et rationalité du quotidien chez les élèves de collège. Thèse de doctorat, Université MONTPELLIER II.

23. Lerouge, A. (2000). La notion de cadre de rationalité. A propos de la droite au collège. Revue Recherche en Didactique des Mathématiques. 20(2). 171-208

24. Maarouf, A et Kouhila, M. (2001). La dynamique élémentaire dans l'enseignement fondamental marocain: analyse des difficultés d'apprentissage de la notion de force. Didaskalia, 18, 41-59.

25. Maingain, A., Dufour, B et Fourez, G. (2002). Approches didactiques de l'interdisciplinarité. Editions De Boek Université. Belgique.

26. Malafosse, D., Lerouge, A et Dusseau, J.M. (2000). Notions de registres et de cadre de rationalité en inter-didactique des mathématiques et de la physique. Tréma, 18,1-11.

27. Malafosse, D., Lerouge, A et Dusseau, J.M. (2001). Etude en interdidactique des mathématiques et de la physique de l'acquisition de la loi d'Ohm au collège: Changement de cadre de rationalité. Didascalia, 18, 61-98.

28. Malafosse, D et Dusseau, J.M. (2001). Vous avez dit : «U=R.I»? Bulletin de l'union des physiciens, 9, 685-705.

29. MALAFOSSE, D. (2002). Pertinence des notions de cadre de rationalité et de registre sémiotique en didactique de la physique. Recherche en Didactique des Mathématique, 22 (1), 31-76

30. Malongua, F., Beaufils, D et Parzysz, B. (2008). Les équations différentielles du premier ordre en physique en terminale $S$ : le lien avec les mathématiques en question. Le Bup, 12(904), 647-666.

31. Malonga, F. (2009). Les équations différentielles à l'interface mathématique-physique : praxéologie et jeux de cadres de rationalité dans les manuels de terminal $S$. Recherche en didactiques des mathématiques, 29(3), 335-357.

32. Meirieu, P. (1991). Le choix d'éduquer. Ethique et pédagogie, Paris, ESF.

33. Ministère de l'Education Nationale et de la Formation Professionnelle, Direction des curricula, Orientations pédagogiques pour l'enseignement des sciences physiques dans le cycle du secondaire collégien, (Mars 2015). Disponible en ligne à 
http://www.men.gov.ma/curricula/doccurricula/cur-colphys_chimie.pdf

34. Ministère de l'Education Nationale et de la Formation Professionnelle, Direction des curricula, Orientations pédagogiques pour l'enseignement des mathématiques dans le cycle du secondaire collégien, (Août 2009). Disponible en ligne à http://www.men.gov.ma/curricula/doccurricula/cur-col-math.pdf

35. Moffet, J.D. (1993). Le transfert: le bon ou le mauvais génie de l'apprentissage. Québec français, 88, 33-35. Disponible en ligne à : http://id.erudit.org/iderudit/44564ac.

36. Mohapatra, J.K. et Bhattacharyya, S. (1989). Pupils, teachers, induced incorrect generalization and the concept of force. International Journal of Science Education, 11(4), 429-436.

37. O'Malley, C., O'Sheat, T et Scanlon, E. (1994). The conception of force and motion of students aged between 10 and 15 years: an interview study designed to guide instruction. International Journal of Science Education, 16 (2), 215-229.

38. Perrenoud, P. (2000). D’une métaphore l'autre: Transférer ou mobiliser ses connaissances. In Dolz, J et Ollagner, E. (dir) (2000). L’énigme de la compétence en éducation, Bruxelles, De Boeck, Coll. Raisons éducatives, 45-60.

39. Presseau, A. (2000). Analyse de l'efficacité d'interventions sur le transfert des apprentissages en mathématiques. Revue des sciences de l'éducation, XXVI (3), 514-544.

40. Raouf.K., Belazzaar, I., Radi, M., Moussetad, $M$ et Talbi Mohammed. (2016). Les difficultés inhérentes à la mobilisation des connaissances mathématiques dans la physique, cas de la mécanique au collège. European Scientific Journal, 12 (25).185- 198

41. Rey, B et Develay, M. (1996). Les compétences transversales en question, Paris, ESF.

42. Robert, C et Treiner, J. (2004). Une double émergence. Bulletin de l'union des physiciens, Paris, France, 867, 1399-1470.

43. Rogalski, M (2013). Modéliser dans la classe de mathématiques ? pourquoi ? Comment? Quelles relations entre Mathématiques et physique ?, Actes CORFEM, 20 ${ }^{\text {ème }}$ colloque, IUF de Grenoble, 13-14 juin 2013.

44. Rutherford, F. J \& Ahlgren, A. (1990). Science for all Americans. New York, NY: Oxford Press. In Prestwich, J, S. "Teacher Definitions of Integration in Primary Grades" (2012) Brigham Young University BYU scholars archive. Paper 3738.

45. Squalli, H., Theis, L., Hasni, A et Benoit, D. (2010). L'interdisciplinarité entre mathématiques et sciences à l'école 
secondaire. Une étude de cas. Actes du congrès Espace Mathématique Francophone-2009, Groupe de travail 5 : Interactions entre mathématiques et autres disciplines dans les formations générales et professionnelles. Sénegal.

46. Tardif, J et Meirieu, Ph. (1996). Stratégie pour favoriser le transfert des connaissances. Vie pédagogique, 98. 4-7.

47. Tardif, J et Presseau, A. (1998). Quelques contributions de la recherche pour favoriser le transfert des apprentissages. Vie pédagogique, 108, 39-44.

48. Tardif, J. (1999). Le transfert des apprentissages. Montréal, les Editions logiques.

49. TIMSS: Trends in International Mathematics and Science Study (1999, 2003, 2007, 2011).

50. Touma, G. (2008). Activité cognitive d'interprétation. Annales de Didactique et de Sciences Cognitives. 13. 93-111.

51. Twigger, D., Byard, M., Driver, R., Drape, S., Hartley, R., Hennessy, S., Ramzan, M.,

52. VIENNOT, L. (1986). Bilans des forces et loi des actions réciproques : analyse des difficultés des élèves et enjeux didactiques. Bulletin de l'Union des Physiciens, 716, 951-97 